\title{
Opposing influences on conflict-driven adaptation in the Eriksen flanker task
}

\author{
JULIE M. BUGG \\ Washington University, St. Louis, Missouri
}

\begin{abstract}
Compatibility effects in conflict paradigms are reduced following incompatible trials, and this effect is referred to as conflict adaptation. A perplexing pattern exists, however, with conflict-driven adaptation emerging in several paradigms (e.g., Stroop, Simon) but not consistently in the Eriksen and Eriksen (1974) flanker task. The present experiments address the seemingly elusive presence of conflict adaptation in this task. Experiment 1 shows that a negative-priming-like slowing may be masking conflict adaptation in the flanker task. In Experiment 2, conflict adaptation was revealed when a larger stimulus set designed to reduce negative priming was implemented. Taken together, the findings indicate that a consideration of processes opposing conflict adaptation in the flanker task may help reconcile prior findings.
\end{abstract}

Over the past several decades, significant progress has been made in understanding the processes that support cognitive control, the goal-oriented coordination of cognitive resources. One major arena in which cognitive control has been investigated is in conflict paradigms, wherein a target response is competing with an opposing response that is habitual or experimentally primed but is incorrect. Of interest in the present article is the reduction in the magnitude of compatibility effects (i.e., the difference in reaction time $[\mathrm{RT}]$ between compatible $[\mathrm{C}]$ trials, wherein the to-be-attended information elicits the same response as the to-be-ignored information, and incompatible [I] trials, wherein competing responses are elicited by the tobe-attended and to-be-ignored information) that occurs following trials involving processing conflicts.

One interpretation of this reduction, the conflict monitoring account, centers on the notion that control is heightened when response conflict is detected on trial $n-1$, thereby helping participants adapt to such conflict on the ensuing trial (i.e., conflict adaptation) (Botvinick, Braver, Barch, Carter, \& Cohen, 2001). Supportive of this account is the RT advantage that is observed on incompatible trials that are preceded by incompatible (II) as opposed to compatible (CI) trials. This interpretation has been challenged, however, by findings that appear to favor a basic priming mechanism over a control mechanism. Current evidence favors the priming mechanism particularly for conflict adaptation effects in the Eriksen flanker task. In this article, we review this evidence and consider both conflict monitoring and priming accounts (see also Egner, 2007, for a review). We then offer an alternative theoretical analysis that proposes an interplay between basic priming and cognitive control mechanisms, and we report two experiments to test this framework.

\section{The Eriksen Flanker Task and Conflict Adaptation}

During the Eriksen flanker task, participants are asked to respond to a target stimulus that is surrounded by flanker stimuli on each side. In a variant of the task, a right- or left-pointing central arrow is surrounded by right- or leftpointing distractor arrows (Eriksen \& Eriksen, 1974). In the incompatible condition (e.g., $<<<><<<$ ), the flanker stimuli point in a direction opposite to the target stimulus; thus, the participant must resolve the conflict between the two potential responses. RTs and error rates tend to be inflated in the incompatible condition relative to a compatible condition (e.g., $<<<<<<<$ ) in which only one response is elicited. Such findings suggest that response conflict is detrimental to performance. Interestingly, though, when performance is examined as a function of both current trial type and previous trial type, it appears that response conflict can, under certain conditions, facilitate performance. Specifically, Gratton, Coles, and Donchin (1992) showed that the speed of responding on incompatible (conflict) trials that immediately followed another incompatible (conflict) trial (i.e., II trials) was faster than on incompatible trials that followed a compatible trial (i.e., CI trials). Subsequent research has confirmed this so-called "conflict adaptation effect" (Botvinick, Nystrom, Fissell, Carter, \& Cohen, 1999; Kerns et al., 2004) and further demonstrated that postincompatible trial adjustments may include decreased error rates (Ullsperger, Bylsma, \& Botvinick, 2005).

\section{Theoretical Interpretations \\ of the Conflict Adaptation Effect}

One account of the conflict adaptation effect is based on the neurobiologically oriented conflict monitoring model 
(Botvinick et al., 2001). According to this model, the anterior cingulate cortex elicits a conflict monitoring signal on trial $n-1$, particularly for cases in which response conflict is present, as in an incompatible trial. This signal is purported to trigger the dorsolateral prefrontal cortex to increase cognitive control and, thereby, reduce one's susceptibility to conflict. Thus, performance on the immediately following incompatible trial (trial $n$ ) benefits from this increase in cognitive control. The work of Kerns et al. (2004) provides direct support for the conflict monitoring account, showing that performance on II trials during a Stroop task not only is faster than on CI trials, but also is associated with significantly less activity in the anterior cingulate. Furthermore, Kerns et al. found that the degree of postconflict adjustment (i.e., adaptation) was directly and positively related to the activation of the dorsolateral prefrontal cortex, supporting the idea that the anterior cingulate's signal modulates control regions' influence on performance.

An alternative account attributes the conflict adaptation effect to stimulus-specific repetition priming. Mayr, Awh, and Laurey (2003) provided initial evidence favoring this account, showing that the pattern of means representative of conflict adaptation (II trials faster than CI trials) was found only for II repetition trials in which the stimulus and response on trial $n-1$ (e.g., $<<<><<<$ ) matched those on trial $n$ (e.g., $<<<><<<$ ), but not on II nonrepetition trials in which both the stimulus and response on trial $n-1$ (e.g., $<<<><<<$ ) differed from those presented on trial $n$ (e.g., $>>><>>>$ ). The conflict adaptation pattern was again absent on nonrepetition trials in a second experiment in which complete repetitions of stimuli and responses were eliminated by alternating between a leftright and up-down flanker task (Mayr et al., 2003). Other labs have also failed to observe the conflict adaptation pattern in the Eriksen flanker task when the facilitative effects of priming have been taken into account. In fact, in spite of considerable efforts to induce greater conflict, to use a range of stimulus materials, and to maximize statistical power, Nieuwenhuis et al. (2006) found no evidence of conflict-driven adaptation effects in the flanker task across five experiments.

The strong implication of these findings is that speeded responding on II trials and, relatedly, conflict adaptation effects are governed by a priming mechanism, not conflictdriven shifts in cognitive control. However, a closer consideration of the literature reveals that conflict adaptation is reliably observed in several other conflict paradigms, consistent with the conflict monitoring account. For instance, conflict adaptation has been observed, irrespective of priming, in Stroop (Egner \& Hirsch, 2005; Kerns et al., 2004; Notebaert, Gevers, Verbruggen, \& Liefooghe, 2006) and Simon tasks (Sohn \& Carter, 2003, as cited by Ullsperger et al., 2005; Wuhr \& Ansorge, 2005). Collectively, these studies dispute the repetition priming account. In spite of this, these studies do not offer a complete explanation of why the conflict adaptation effect is generally absent on nonrepetition trials of the Eriksen flanker task.

One hypothesis is that a conflict-driven control process simply does not benefit performance above and beyond repetition priming in the flanker paradigm. Disfavoring this hypothesis is the single report of a conflict adaptation effect on nonrepetition trials of the Eriksen flanker task (Ullsperger et al., 2005). Also disfavoring this hypothesis, two reports show evidence of conflict adaptation using a numerical version of the Eriksen flanker task, even when repetition trials were eliminated from the analyses (Notebaert \& Verguts, 2006; Ullsperger et al., 2005).

A second, more viable, hypothesis is that task parameters may preclude obtainment of conflict adaptation effects. In particular, the limited size of the stimulus and response set in standard versions of the Eriksen flanker task may be related to the elimination of the conflict adaptation effect. In support of this hypothesis, when the flanker task is modified such that the stimulus set is expanded beyond two stimuli and responses (e.g., the numerical flanker task with nine stimuli/responses, Notebaert \& Verguts, 2006; Ullsperger et al., 2005; a colored bar flanker task with six stimuli and three responses, Verbruggen, Notebaert, Liefooghe, \& Vandierendonck, 2006), conflict-driven adaptation effects are observed (but see Wendt, Heldmann, Munte, \& Kluwe, 2007, for an exception).

Why might the size of the stimulus and response set be a critical determinant of the presence or absence of conflict adaptation? The size of these sets may influence the degree to which a negative-priming-like mechanism slows performance on certain trial sequences in the Eriksen flanker task (for similar arguments, see, e.g., Notebaert \& Verguts, 2006; Ullsperger et al., 2005). Consider, for example, an II nonrepetition trial on which " $<<<><<<$ " is presented on trial $n-1$. If processing of the central arrow is accompanied by inhibition of the flankers and the associated left keypress response, then the left keypress response to " $\rangle\rangle\rangle\langle\rangle\rangle\rangle$ " on trial $n$ may be disproportionately slowed because the participant must overcome the inhibition from the preceding trial in order to produce the correct response. Stadler and Hogan (1996) established that such slowing, which they referred to as negative priming (Neill, 1977; Tipper, 1985), is particularly apparent on complete reversal nonrepetition trials in the Eriksen flanker task. In Stadler and Hogan's version of the task, trials consisted of and were analyzed as prime/probe pairs. On complete reversal nonrepetition trials, the attended (target) information in the prime stimulus became the ignored (flanker) information in the probe stimulus, and the ignored information in the prime became the attended information in the probe.

Importantly, $100 \%$ of the transitions on II nonrepetition trials are complete reversals when the flanker task involves just two stimuli and responses, as in prior studies finding no evidence of conflict-driven adaptation (Mayr et al., 2003; Nieuwenhuis et al., 2006). In contrast, when the size of the stimulus and response set is increased, the percentage of nonrepetition trials that are complete reversals is reduced. Correspondingly, negative priming may also be attenuated, thereby maximizing one's ability to observe conflict-driven adaptation.

In this article, we expand on these theoretical dynamics and report two experiments that explore whether opposing processes such as negative priming may mask the conflict adaptation effect when particular task parameters are 
present. In Experiment 1, we first demonstrate a negativepriming-like slowing and the absence of conflict adaptation during II nonrepetition trials in the flanker task using the methodology of Mayr et al. (2003). In Experiment 2, we introduce a version of the flanker task in which the influence of negative priming is attenuated by increasing the size of the stimulus set such that complete nonrepetition trials are eliminated, and demonstrate the return of the conflict adaptation effect on II nonrepetition trials.

\section{EXPERIMENT 1}

The purpose of Experiment 1 was twofold. First, we sought to examine whether the finding of the conflict adaptation pattern on repetition but not nonrepetition trials (Mayr et al., 2003; Nieuwenhuis et al., 2006) was replicable, and if it was, to examine the extent to which negative priming may be differentially slowing performance on nonrepetition trial sequences. Therefore, the major methodological features from Experiment 1 of Mayr et al. were replicated in the present experiment. To gain evidence for the negative priming explanation, the primary modification to Mayr et al.'s method was the introduction of neutral trials. Neutral trials were composed of a central right- or left-facing arrow surrounded by asterisks. As such, the neutral trials were similar to incompatible trials with regard to the presence of perceptual conflict. The key difference between neutral and incompatible trials relates to the presence of response conflict. Because the asterisks were not assigned a response key, we expected that response conflict and any corresponding inhibition of the flankerinduced response would not be present on neutral trials. Thus, including neutral trials provided a baseline against which to estimate the lingering effects of inhibiting the flanker-induced response on incompatible trials.

The primary set of predictions concerns the conflict adaptation pattern. Table 1 shows the theoretical processes underlying these predictions. If the conflict adaptation pattern is due solely to priming dynamics, then speeded responding should be observed on II repetition trials (e.g., $>>><>>>,>>><>>>$ ) relative to baseline CI repetition trials (e.g., $<<<<<<<,>>><>>>$ ), but not on II nonrepetition trials relative to CI nonrepetition trials. The idea here is that a complete repetition of both stimuli and responses from trial $n-1$ to trial $n$, as in the II repetition case, should result in substantial positive priming relative to a trial-to-trial transition in which only the response repeats, as in the CI repetition case. Alternatively, if conflict adaptation is due to cognitive control adjustments in response to conflict monitoring, then the conflict adaptation pattern should be observed on both II repetition and II nonrepetition trials relative to baseline CI trials. This is because conflict is present on trial $n-1$ in both cases.

Our account assumes that priming dynamics will also include negative priming. Prior work by Stadler and Hogan (1996) is especially informative with regard to the predicted slowing that is expected to take place on particular trial types because of negative priming. As described above, they found that performance on the Eriksen flanker task was especially slowed for complete reversal nonrepetition II trials (e.g., $<<<><<<,>>><>>>$ ). They also observed slowing on trials in which the to-beinhibited response was recently activated, as in the case of CI nonrepetition trials (e.g., $>>>>>>>\rangle\rangle\rangle,\langle>\rangle\rangle$ ) (see also Neumann \& DeSchepper, 1991, Experiment 3, for a similar result in a more typical negative priming paradigm). Stadler and Hogan used the term "negative priming" to refer to both instances of slowing, although the two instances differed appreciably in magnitude, with the former leading to greater slowing than the latter, relative to neutral trials. ${ }^{1}$ Accordingly, in the present experiment, we expected that negative priming dynamics alone would produce slower performance on II nonrepetition trials than on CI nonrepetition trials, a reversal or elimination of the conflict adaptation pattern.

Table 1

Theoretical Processes Underlying Predictions Concerning Conflict Adaptation Effects for Key Trial-to-Trial Transitions

\begin{tabular}{|c|c|c|c|c|c|c|}
\hline & & \multicolumn{2}{|c|}{ Trial-to-Trial Transitions } & \multicolumn{3}{|c|}{ Presumed Theoretical Processes } \\
\hline & & Previous Trial & Current Trial & Priming & Adaptation & NP \\
\hline \multirow[t]{3}{*}{ Repetition } & NI & $* * *<* * *$ & $>>><>>>$ & & & \\
\hline & $\mathrm{CI}$ & $<<<<<<<$ & $>>>\langle>\rangle\rangle$ & & & \\
\hline & II & $>>><>>>$ & $>>>\langle>\rangle>$ & + & + & \\
\hline \multirow[t]{3}{*}{ Nonrepetition } & NI & $* * *>* * *$ & $>>><>\rangle>$ & & & \\
\hline & $\mathrm{CI}$ & $>>>>>>$ & $>>\rangle\langle>\rangle$ & & & - \\
\hline & II & $<<<><<<$ & $>\rangle>\langle>\rangle\rangle$ & & + & - \\
\hline
\end{tabular}

Note-NI, a trial sequence in which an incompatible trial follows a neutral trial; CI, a trial sequence in which an incompatible trial follows a compatible trial; II, a trial sequence in which an incompatible trial follows an incompatible trial. For repetition trials, either the response alone (e.g., NI, CI) or both the stimulus and response repeated (e.g., II). A "+" in the priming column indicates that speeded responding is expected on a given trial type (e.g., II repetition) relative to a baseline condition (e.g., CI or NI repetition) on account of positive priming. A "+ " in the adaptation column indicates an expected speeding of RTs on a given trial type (e.g., II repetition or II nonrepetition) relative to a baseline condition (e.g., CI repetition or nonrepetition, respectively) on account of conflict adaptation. A "-" in the negative priming (NP) column represents an expected slowing of RTs on a given trial type (e.g., II nonrepetition) relative to a baseline condition (e.g., NI nonrepetition). The thicker "-" implies greater slowing. 
Our account, however, assumes that the conflict adaptation pattern is dependent not only on priming dynamics, but also on conflict monitoring. Our account assumes that the speeded responding on II relative to CI repetition trials reflects both positive priming and conflict monitoring. The critical departure point distinguishing our account from the priming-only account hinges on nonrepetition trials. Whereas the priming-only account predicts that conflict adaptation will never be observed on nonrepetition trials, our account suggests that conflict adaptation will be observed on these trials under particular task conditions (as revealed in Experiment 2). That is, our account anticipates that observation of the conflict adaptation effect on nonrepetition trials is dependent on the dynamic interplay of the theoretical processes depicted in Table 1. To develop the fruitfulness of this account, Experiment 1 first reveals these theoretical processes. One process, negative priming, is expected to slow performance on trials of both the II and CI nonrepetition types, with greater slowing for the former trial type (Stadler \& Hogan, 1996). A second process, conflict adaptation, is expected to speed performance, but only on the II trials. Thus, for trial-to-trial transitions of the II nonrepetition type, negative priming and conflict adaptation are acting in opposition to one another. Two important consequences are anticipated. Because the conflict adaptation process does not completely mitigate the negative priming process, a first prediction is that a negative priming effect for II nonrepetition trials should remain. A second consequence is that the magnitude of slowing on II nonrepetition trials is not as extreme as it would be if negative priming alone were operative. Therefore, our second prediction is that RTs on II nonrepetition trials will be similar to those exhibited on CI trials (trials on which negative priming is less robust, as noted above).

\section{Method}

Participants. Seventy-five Colorado State University undergraduates participated, in partial fulfillment of course credit. Informed consent was obtained and participants were screened for handedness, neurological and/or psychiatric illnesses (such as ADHD), and history of significant head trauma. Data from 13 participants who were left-handed or who reported the presence of either illness or head trauma were excluded from further analysis.

Design. The study employed a $3 \times 3$ within-subjects design within an Eriksen flanker task. Previous trial type (compatible [C] vs. neutral $[\mathrm{N}]$ vs. incompatible $[\mathrm{I}]$ ) and current trial type (compatible $[\mathrm{C}]$ vs. neutral $[\mathrm{N}]$ vs. incompatible [I]) represented the factors. Compatible trials were composed of a central display with seven right-facing or seven left-facing arrows in a row. Incompatible trials were composed of a central display of seven arrows, wherein the central-facing arrow (left or right) pointed in the opposite direction to the flanker arrows (right or left, respectively). Neutral trials were composed of a central right- or left-facing arrow, surrounded by three asterisks on each side. Given that the asterisks were never associated with a response key, the neutral condition was not expected to elicit response conflict. The central arrow faced left on $50 \%$ of the $\mathrm{C}, \mathrm{N}$, and I trials and faced right on the other half of the trials. The stimulus display was $15^{\circ}$ wide, and individual stimuli were $1^{\circ}$ high and wide.

Procedure. The experiment was conducted using E-Prime (Psychology Software Tools, Inc., Pittsburgh, PA). During the practice phase, participants were instructed to press the left ("h") or right (“j”) response key on a keyboard, using left and right index fingers, respectively, depending on the direction that the central arrow in the stimulus display was pointing. Participants were instructed to respond as quickly and accurately as possible. Twenty-four practice trials were presented, during which each of the trial types $(\mathrm{C}, \mathrm{I}, \mathrm{N})$ appeared equally often.

Following practice, the test phase began. The test consisted of eight blocks of 72 trials with equal numbers of $\mathrm{C}$, I, and $\mathrm{N}$ trials within each block. Eight CC, CN, CI, NC, NN, NI, IC, IN, and II trials were presented in each block. During the task, the stimulus remained on-screen until a response was made, and the response-tostimulus interval (RSI) was set at $1,000 \mathrm{msec}$, replicating Mayr et al. (2003; U. Mayr, personal communication, March 31, 2004). Also following Mayr et al., a fixation cross was presented beneath the central arrow on stimulus screens and remained during the otherwise blank RSI. Participants were reminded of the instructions following the completion of each block and were debriefed following completion of the task. The dependent measures of interest were RT (in milliseconds) and error rate.

\section{Results}

Following Mayr et al. (2003), trials following errors and error trials were excluded from the RT analyses, and trials following errors were excluded from the error rate analyses. Because the primary predictions in this study dealt with a contrast between repetition and nonrepetition trials, we coded transition type (target repetition vs. target nonrepetition) and treated it as a separate factor in a three-way within-subjects ANOVA for both dependent measures. The additional factors were previous trial type (compatible vs. neutral vs. incompatible) and current trial type (compatible vs. neutral vs. incompatible). The alpha level was set at .05 for this and all subsequent analyses presented in this article.

Reaction time. The three-way interaction of transition type, previous trial type, and current trial type was significant $\left[F(4,244)=14.59, M S_{\mathrm{e}}=1,687.01\right]$. The standard flanker (i.e., compatibility) effect was evident, because incompatible trials $(M=582)$ were slower than neutral trials $(M=467)$, which were slower than compatible trials $(M=462)(t \mathrm{~s} \geq 3.08)$. Because the primary predictions involved a differentiation between repetition and nonrepetition trials, separate two-way ANOVAs were conducted to explore the critical interaction between previous and current trial type. For repetition trials, this interaction was significant $\left[F(4,244)=22.28, M S_{\mathrm{e}}=2,408.71\right]$. Replicating Mayr et al. (2003), the pattern of means consistent with the conflict adaptation effect was observed (see Figure 1$)$. RT on the II trials $(M=543)$ was significantly faster than RT on the CI trials $(M=593)[F(4,244)=$ $\left.32.54, M S_{\mathrm{e}}=2,408.71\right]$. The compatibility effect was reduced from $160 \mathrm{msec}$ following compatible trials to $63 \mathrm{msec}$ following incompatible trials.

In contrast, for nonrepetition trials, the two-way interaction of current and previous trial type was not significant $[F(4,244)=1.86, p=.12$; see Figure 1], further replicating Mayr et al. (2003). Of particular interest was examining whether negative priming emerged during performance of II nonrepetition trials, because the present account posits that negative priming may be masking conflict adaptation on these trials. In line with the observed null interaction, there was a significant negative 


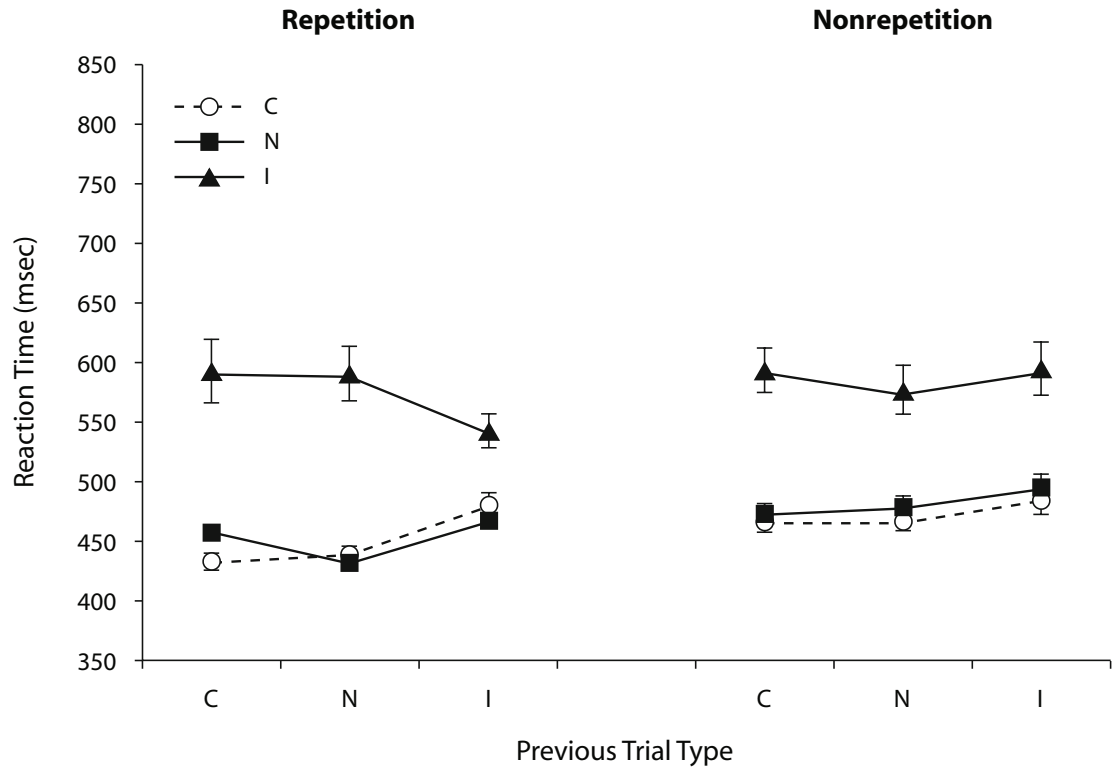

Figure 1. Mean reaction time (in milliseconds) for repetition and nonrepetition trials in Experiment 1 as a function of previous and current trial type. Error bars represent the standard error of the mean.

priming effect such that II nonrepetition trials $(M=595)$ were $18 \mathrm{msec}$ slower than NI nonrepetition trials $(M=$ 577), a difference confirmed by a planned comparison $\left[F(4,244)=8.39, M S_{\mathrm{e}}=1,270.07\right]$. As anticipated, the CI nonrepetition trials $(M=594)$ were also significantly slower than the NI nonrepetition trials $[F(4,244)=6.82$, $\left.M S_{\mathrm{e}}=1,270.07\right]$. Note that similar RTs were observed for II and CI nonrepetition trials $(F<1)$, consistent with the present account (as discussed below).

Error rate. Error rates are presented in Table 2. The three-way interaction of transition type, previous trial type, and current trial type was also significant for error rate $\left[F(4,244)=3.75, M S_{\mathrm{e}}=.001\right]$. Consistent with the RT analyses, the follow-up two-way ANOVAs indicated that the previous trial type $\times$ current trial type interaction was significant for repetition trials $[F(4,244)=4.66$, $\left.M S_{\mathrm{e}}=.001\right]$ but not for nonrepetition trials $[F(4,244)=$ $1.23, p=.30]$. For repetition trials, the error rate was significantly lower on II trials $(M=.05)$ than on CI trials $(M=.07)$, mirroring the results for RT $[F(4,244)=8.24$, $\left.M S_{\mathrm{e}}=.001\right]$. For the nonrepetition trials used to test for negative priming (II, NI, and CI), only the error rates for II $(M=.05)$ and $\mathrm{CI}(M=.04)$ trials differed $[F(4,244)=$ $\left.3.10, M S_{\mathrm{e}}=.001\right]$.

\section{Discussion}

In line with the repetition priming account, the pattern of means reflective of the conflict adaptation effect was observed on repetition, but not on nonrepetition trials, replicating Mayr et al. (2003). The lack of the conflict adaptation pattern on nonrepetition trials is unanticipated by the conflict monitoring account. By this account, conflict adaptation should have been observed for both types of II trials since conflict is present on trial $n-1$ in each case. As predicted by the present account, the RT on II nonrepetition trials was significantly slower than the RT on NI nonrepetition trials. This demonstration of a negative priming effect lends support to the notion that negative priming may be working in opposition to conflict adaptation during II nonrepetition trials. We acknowledge that caution is warranted in interpreting the slowing on II trials as negative priming, per se, because a similar magnitude of slowing was also observed on IN and IC nonrepetition trials relative to $\mathrm{NN}$ and $\mathrm{NC}$ trials (see Figure 1), respectively. It is uncertain whether other unanticipated factors are contributing to the slowing on these trials or possibly on II trials. The key point for present purposes, however, is that a particular dynamic whereby one process acts to slow performance while a second simultaneously acts to speed performance exists on II nonrepetition trials but does not exist on II repetition trials.

We suggest that this dynamic underlies two results. First, as is typical in the flanker paradigm, the conflict adaptation pattern was not observed on II nonrepetition trials. Second, the RT on II and CI nonrepetition trials did not differ. Negative priming dynamics alone would not predict this equivalence (Neumann \& DeSchepper, 1991; Stadler \& Hogan, 1996). This equivalence is expected, however, if one assumes that conflict monitoring speeded performance to some degree on the II (but not the CI) trials.

This pattern of findings has important implications for existing knowledge and theoretical interpretations of conflict adaptation effects. First, it indicates that opposing 
Table 2

Mean Error Rates, With Standard Deviations, for Repetition and Nonrepetition Trials As a Function of Trial Sequence in Experiment 1 and Experiment 2

\begin{tabular}{|c|c|c|c|c|c|c|c|c|c|c|c|c|c|c|c|c|c|c|}
\hline \multirow[b]{3}{*}{ Study } & \multicolumn{18}{|c|}{ Trial Sequence } \\
\hline & \multicolumn{2}{|c|}{$\mathrm{CC}$} & \multicolumn{2}{|c|}{$\mathrm{CN}$} & \multicolumn{2}{|c|}{$\mathrm{CI}$} & \multicolumn{2}{|c|}{$\mathrm{NC}$} & \multicolumn{2}{|c|}{$\mathrm{NN}$} & \multicolumn{2}{|c|}{ NI } & \multicolumn{2}{|c|}{ IC } & \multicolumn{2}{|c|}{ IN } & \multicolumn{2}{|c|}{ II } \\
\hline & $M$ & $S D$ & $M$ & $S D$ & $M$ & $S D$ & $M$ & $S D$ & $M$ & $S D$ & $M$ & $S D$ & $M$ & $S D$ & $M$ & $S D$ & $M$ & $S D$ \\
\hline \multicolumn{19}{|l|}{ Experiment 1} \\
\hline Repetition & .01 & .02 & .01 & .03 & .07 & .06 & .01 & .02 & .01 & .02 & .08 & .07 & .01 & .01 & .01 & .03 & .05 & .06 \\
\hline Nonrepetition & .02 & .03 & .02 & .03 & .04 & .06 & .01 & .02 & .02 & .03 & .05 & .07 & .02 & .03 & .02 & .03 & .05 & .07 \\
\hline \multicolumn{19}{|l|}{ Experiment 2} \\
\hline Repetition & .01 & .01 & & & .06 & .05 & & - & & & & & .01 & .03 & & & .04 & .05 \\
\hline Nonrepetition & .07 & .05 & & - & .05 & .03 & & - & & & & & .04 & .03 & & & .07 & .04 \\
\hline
\end{tabular}

Note-CC, congruent, congruent; CN, congruent, neutral; CI, congruent, incongruent; NC, neutral, congruent; NN, neutral, neutral; NI, neutral, incongruent; IC, incongruent, congruent; IN, incongruent, neutral; II, incongruent, incongruent.

processes may underlie performance on II trials in the Eriksen flanker task, particularly complete reversal nonrepetition II trials. Both Mayr et al. (2003) and Nieuwenhuis et al. (2006), who failed to observe conflict adaptation on nonrepetition trials, used paradigms in which the II nonrepetition trials were of this type. Our results suggest that negative priming may have masked conflict adaptation in these studies. Second, the size of the conflict adaptation effect is often small, and in some cases smaller than the 18-msec negative priming effect observed in Experiment 1 (see, e.g., Ullsperger et al., 2005). Therefore, an important implication of the present view is that stronger evidence of conflict adaptation would be more consistently observed on nonrepetition trials under conditions that reduce the influence of negative priming. We conducted Experiment 2 to test this possibility.

\section{EXPERIMENT 2}

A stimulus set consisting of left, right, up, and down arrows was used, and each of two stimuli (e.g., a right arrow and a down-facing arrow) was assigned to one response key (e.g., the right key). The inclusion of just two additional stimuli greatly expands the space of possible trialto-trial transitions that can occur on nonrepetition trials. Of the transitions on nonrepetition trials, $100 \%$ are complete reversals when the flanker task involves just two stimuli, as in Experiment 1 (see also Mayr et al., 2003, Experiment 1; Nieuwenhuis et al., 2006). With this larger stimulus set, only $25 \%$ of the possible transitions on nonrepetition trials are complete reversals. Thus, in Experiment 2, we were able to use a version of the flanker task that excluded complete reversal nonrepetition trials (which have previously been associated with the strongest degree of negative priming) and still have II nonrepetition trial transitions to evaluate the presence of conflict adaptation.

As in Experiment 1, the repetition priming account predicts that the conflict adaptation effect should be observed on repetition but not on nonrepetition trials. In contrast, because negative priming is expected to be attenuated by the expanded stimulus set and the elimination of complete nonrepetition trials, the present account predicts that the conflict adaptation effect will be observed on both repetition and nonrepetition trials. To foreshadow, the manipulation was effective in revealing conflict adaptation on repetition and nonrepetition trials. This result motivated a subsequent set of analyses in which we compared the magnitude of the conflict adaptation effect for high-conflict nonrepetition trials (involving horizontally oriented flankers; e.g., $<<<><<<$ ) and low-conflict nonrepetition trials (involving vertically oriented flankers; e.g., $M \wedge>\wedge$ ). Some past studies have found a positive relationship between the degree of conflict on trial $n-1$ and the magnitude of adaptation on trial $n$ (e.g., Kerns et al., 2004). Others, however, have not (Nieuwenhuis et al., 2006). Theoretically, conflict monitoring theory would anticipate an effect of the degree of conflict. Thus, this issue warrants further evaluation.

\section{Method}

Participants. Forty-seven Colorado State University undergraduates participated, in partial fulfillment of course credit. Informed consent was obtained from each participant. Participants were screened as in Experiment 1, and data from 10 participants who were left-handed or who reported the presence of either illness or head trauma were excluded from further analysis.

Design. The study employed a $2 \times 2$ within-subjects design within an Eriksen flanker task. Previous trial type (compatible [C] vs. incompatible [I]) and current trial type (compatible [C] vs. incompatible [I]) represented the factors. Compatible trials were composed of a central display with seven arrows facing the same direction, whereas incompatible trials were composed of seven arrows in a row, with the central arrow corresponding to the response that was opposite the flanker arrows.

Because the left- and up-facing arrows were both assigned to the left response key, and the right- and down-facing arrows were both assigned to the right response key, incompatible trials were composed of either a left- or up-facing arrow surrounded by right- or down-facing arrows, or a right- or down-facing arrow surrounded by left- or up-facing arrows. Each incompatible stimulus arrangement was presented seven times within a block of trials. There were four stimulus arrangements used on compatible trials, one each pertaining to right-, left-, up-, and down-facing arrows. In order to avoid the potential for stimulus-based conflict (see, e.g., Verbruggen et al., 2006), all arrows pointed in exactly the same direction on compatible trials. Each compatible stimulus arrangement was used 14 times within a block. Therefore, $50 \%$ of the trials were compatible and $50 \%$ of the trials were incompatible. Also, $50 \%$ of the compatible and incompatible trials required a left keypress in response to the central target and $50 \%$ required a right keypress response. Of interest was the interaction of the two independent variables, previous and current trial type. Within each experimental block, a sequence of trial types was pseudorandomly generated such that an equivalent 
number (28) of CC, CI, II, and IC trials were presented. To attenuate the influence of negative priming on II trials, complete reversal nonrepetition sequences (e.g., $<<<><<<,>>><>>$ ) were not permitted to occur.

Procedure. A training phase was used to familiarize participants with the appropriate stimulus-response key assignments. Participants responded to individual arrows presented one at a time in the center of the screen. Twelve right-, left-, up-, and down-facing arrows were randomly presented, for a total of 48 trials. Participants were instructed to press the left key using their left index finger if the arrow pointed left or up, and to press the right key using their right index finger if the arrow pointed right or down. Trials did not advance until the correct response was provided.

Following the training phase, a practice phase began. Participants read self-paced instructions paralleling those provided in Experiment 1 , with the exception that participants were now instructed to press the left key if the central arrow pointed left or up and the right key if the arrow pointed right or down. Twenty-four practice trials were presented.

Following practice, the test component consisted of five blocks of 112 trials, emphasizing both speed and accuracy. Blocks were self-paced. During the task, the stimulus remained on-screen until a response was made and the RSI was set at $1,000 \mathrm{msec}$, replicating Mayr et al. (2003) and Experiment 1. Similarly, a fixation cross was presented beneath the central arrow on stimulus screens and remained on-screen during the otherwise blank RSI. Participants were debriefed following completion of the task. The dependent measures of interest were RT (in milliseconds) and error rate.

\section{Results}

As in Experiment 1, we excluded trials following errors from both the RT and error rate analyses, and error trials from the RT analyses. A three-way within-subjects ANOVA was conducted for both dependent measures. The factors were transition type (target repetition vs. target nonrepetition), previous trial type (compatible vs. incompatible), and current trial type (compatible vs. incompatible).

Reaction time. The three-way interaction of transition type, previous trial type, and current trial type was significant $\left[F(1,36)=6.71, M S_{\mathrm{e}}=2,163.85\right]$. The standard flanker (i.e., compatibility) effect was evident, because incompatible trials $(M=698)$ were slower than compatible trials $(M=570)\left[F(1,36)=40.70, M S_{\mathrm{e}}=29,389.29\right]$. To examine the conflict adaptation pattern on repetition and nonrepetition trials, separate follow-up two-way ANOVAs were conducted. The previous trial type $\times$ current trial type interaction was significant for the repetition trials $\left[F(1,36)=29.84, M S_{\mathrm{e}}=5,518.39\right.$; see Figure 2]. RT was significantly faster on II trials $(M=630)$ in comparison with CI trials $(M=716)\left[F(1,36)=24.73, M S_{\mathrm{e}}=\right.$ $5,518.39]$. The magnitude of the compatibility effect was reduced from $217 \mathrm{msec}$ following compatible trials to $84 \mathrm{msec}$ following incompatible trials.

Most notably, the two-way interaction of previous trial type and current trial type was also significant for nonrepetition trials $\left[F(1,36)=21.20, M S_{\mathrm{e}}=2,611.96\right.$; see Figure 2]. Consistent with the conflict monitoring account, and in line with the predictions of the present account, a strong conflict adaptation effect was observed. II trials were $50 \mathrm{msec}$ faster than CI trials, a difference confirmed by planned comparison $[F(1,36)=18.26$, $\left.M S_{\mathrm{e}}=2,611.96\right]$. The magnitude of the compatibility effect was reduced from $143 \mathrm{msec}$ following compatible trials to $65 \mathrm{msec}$ following incompatible trials. Importantly, this finding implies that conflict adaptation can be observed on nonrepetition trials in the flanker task. Although this finding additionally demonstrates that such adaptation cannot be accounted for by stimulus-specific repeti-
Repetition

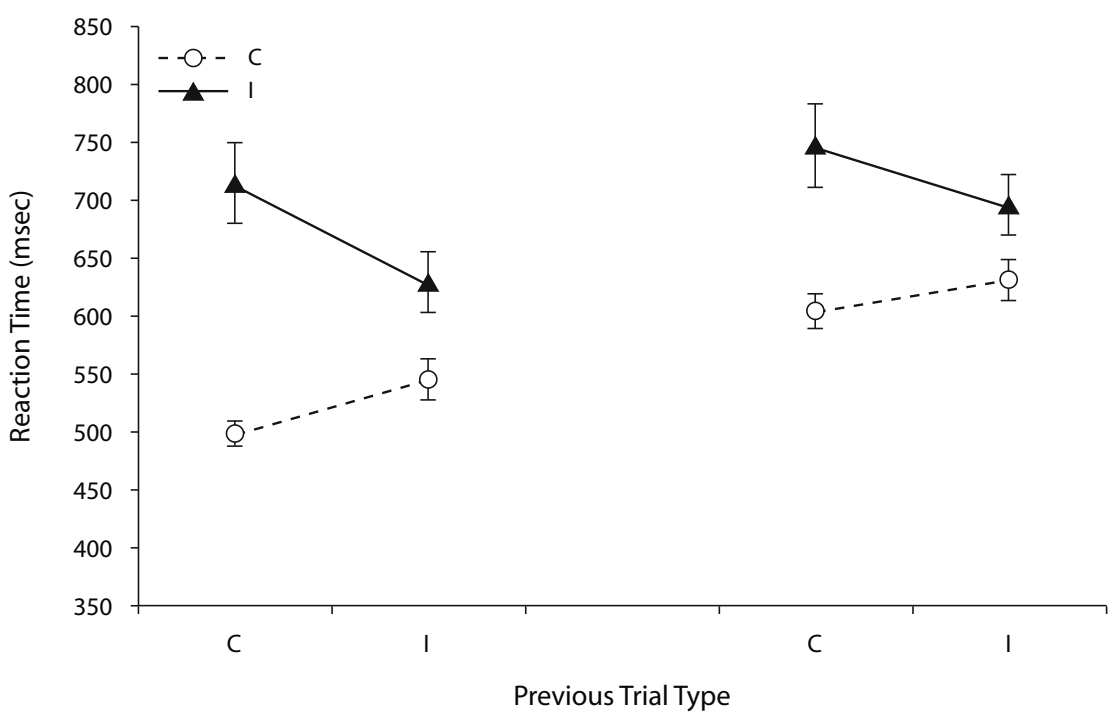

Figure 2. Mean reaction time (in milliseconds) for repetition and nonrepetition trials in Experiment 2 as a function of previous and current trial type. Error bars represent the standard error of the mean. 
tion priming, an important question concerns the degree to which feature integration effects more broadly (e.g., flanker repetitions, target-to-flanker repetitions, flankerto-target repetitions) might account for the adaptation effect observed here (see, e.g., Hommel, Proctor, \& Vu, 2004). ${ }^{2}$ For instance, May, Kane, and Hasher (1995) have shown that RT is speeded when the target on trial $n-1$ becomes the flanker on trial $n$. Likewise, distractor (e.g., flanker) repetitions have been shown to speed responding (e.g., Neumann \& DeSchepper, 1991). Thus, to evaluate this question, the previous trial type $\times$ current trial type interaction was examined for nonrepetition trials after excluding trial-to-trial transitions involving feature repetitions. When the analysis was limited in the strictest possible fashion to such trials (e.g., $>>>\langle>>>$, MMVM $)$ ), the interaction remained significant $[F(1,36)=6.77$, $\left.M S_{\mathrm{e}}=3,297.90\right]$. Most critically, RT was significantly faster on II trials $(M=713)$ than on CI trials $(M=748)$ $\left[F(1,36)=6.91, M S_{\mathrm{e}}=3,297.90\right]$. This finding further confirms that the adaptation effect on nonrepetition trials involves mechanisms (e.g., cognitive control) other than the facilitative effects of bottom-up priming.

To evaluate whether the degree of conflict affected the degree of adaptation, as revealed by the magnitude of the compatibility effect, a two-way within-subjects ANOVA was conducted for the nonrepetition trials, again excluding trial-to-trial transitions involving feature repetitions. The factors were previous trial type (compatible vs. incompatible high conflict vs. incompatible low conflict) and current trial type (compatible vs. incompatible high conflict vs. incompatible low conflict). The main effect of current trial type $\left[F(2,72)=25.16, M S_{\mathrm{e}}=32,055.05\right]$ was significant, with dependent $t$ tests confirming that incompatible high-conflict $(M=771)$ trials were slower than incompatible low-conflict trials $(M=677)$, which were slower than compatible trials $(M=601 ; t \mathrm{~s} \geq 4.04)$. The two-way interaction was also significant $[F(4,144)=$ 13.56, $\left.M S_{\mathrm{e}}=22,137.11\right]$. Most critically, the magnitude of the compatibility effect (the average RT for high- and low-conflict incompatible trials minus the RT for compatible trials) was $156 \mathrm{msec}$ following compatible trials, $59 \mathrm{msec}$ following incompatible high-conflict trials, and $154 \mathrm{msec}$ following incompatible low-conflict trials. The size of the compatibility effect was significantly smaller following incompatible high-conflict relative to incompatible low-conflict trials or compatible trials ( $t \mathrm{~s} \geq 3.70$ ).

Error rate. Error rates are presented in Table 2. Mirroring the RT analysis, the three-way ANOVA was significant for error rate $\left[F(1,36)=20.60, M S_{\mathrm{e}}=.001\right]$. The followup two-way ANOVA approached significance for the repetition trials $[F(1,36)=3.53, p=.07]$. For nonrepetition trials, the two-way interaction of previous and current trial type was significant $\left[F(1,36)=20.77, M S_{\mathrm{e}}=.001\right]$. The error rates were higher on II trials $(M=.07)$ than on $\mathrm{CI}$ trials $(M=.05)\left[F(1,36)=4.74, M S_{\mathrm{e}}=.001\right]$.

When the nonrepetition trial analysis was limited to trials on which feature repetitions were not present, a similar pattern was obtained. The two-way interaction was significant $\left[F(1,36)=10.62, M S_{\mathrm{e}}=.002\right]$, and error rates were higher on II trials $(M=.07)$ than on CI trials $(M=$
.04) $\left[F(1,36)=5.83, M S_{\mathrm{e}}=.002\right]$. Because this finding raises concern regarding a speed-accuracy trade-off, the analysis was conducted again after identifying and eliminating 3 participants with the most discrepant error rates in the CI and II conditions. This resulted in a sample for which error rates were matched in the CI $(M=.95)$ and II $(M=.94)$ conditions $[t(33)=1.63, p=.11]$. The twoway ANOVA indicated that both the previous trial type $\times$ current trial type interaction $\left[F(1,33)=6.40, M S_{\mathrm{e}}=\right.$ $2,476.86]$ and the RT advantage for II $(M=704)$ over CI trials $(M=743)\left[F(1,33)=10.17, M S_{\mathrm{e}}=2,476.86\right]$ remained significant.

As for the effect of the degree of conflict on error rates on the nonrepetition trials, the three-way ANOVA indicated that the previous trial type $\times$ current trial type interaction was significant $\left[F(4,144)=2.91, M S_{\mathrm{e}}=.008\right]$. However, the magnitude of the compatibility effect was larger following incompatible high-conflict $(M=.07)$ relative to incompatible low-conflict $(M=.01)$ or compatible $(M=.003)$ trials. The pattern of reduced compatibility effects in RT but increased compatibility effects in error rates following incompatible high-conflict trials remained when the analyses were restricted to the sample for which error rates on CI and II trials were matched.

\section{Discussion}

The purpose of Experiment 2 was to examine the conflict adaptation pattern on repetition and nonrepetition trials using a larger stimulus set that eliminated the occurrence of complete reversal nonrepetition trials, and any resultant negative priming. Unlike in Experiment 1, the conflict adaptation pattern was observed on both repetition and nonrepetition trials. Importantly, this pattern held when feature integration effects were taken into account on nonrepetition trials. That is, II trials were significantly faster than CI trials even when trial-to-trial transitions did not involve target, flanker, target-to-flanker, or flankerto-target repetitions. This finding is problematic for the repetition priming account and, more broadly, the feature integration account, but is completely in line with the present account. Specifically, the observed conflict adaptation pattern on nonrepetition trials that excluded feature repetitions strongly supports the notion that speeded responding on II trials reflects processes such as heightened cognitive control triggered by conflict monitoring (Botvinick et al., 2001).

The obtainment of conflict adaptation for RT on nonrepetition trials during an Eriksen flanker task is of theoretical significance. Ullsperger et al. (2005) also showed that conflict adaptation effects occur even when repetition trials are completely eliminated during both a standard Eriksen flanker task and a digit flanker task involving a larger stimulus and response set (i.e., digits 1 through 9 on a keypad). What is novel about the present finding is the obtainment of conflict adaptation using a methodology that almost entirely preserves the procedure implemented by Mayr et al. (2003, Experiment 1), a procedure that eliminated adaptation effects on nonrepetition trials. For example, in both studies, the stimulus remained on-screen until a response was made, the RSI was $1,000 \mathrm{msec}$, and 
both speed and accuracy were emphasized. In contrast, the procedure used by Ullsperger et al. differed on several dimensions from Mayr et al. Targets were briefly presented for $100 \mathrm{msec}$ or less, the intertrial intervals (ITIs) were of a longer duration $(3,500 \mathrm{msec}$, minimum $)$, and a speeded task was used (see also Gratton et al., 1992). Additionally, the flanker onset preceded target onset in the standard flanker task experiment (Ullsperger et al., 2005). These dissimilarities make it difficult to infer why conflict adaptation has not been ubiquitously observed on nonrepetition trials in previous studies that utilized flanker tasks. The factor that distinguishes the present experiment from that of Mayr et al. is the size of the stimulus set, which allowed for the elimination of complete nonrepetition II trials, a factor that we believe, in part on the basis of the results of Experiment 1, has an impact on the obtainment of conflict adaptation by way of its influence on the magnitude of negative priming.

At odds with the interpretation that conflict-triggered control adjustments underlie the RT pattern, however, were the error rates on the nonrepetition trials. Heightened cognitive control might be expected to lead not only to speeded but also to more accurate responding. Yet the error rate was $2 \%$ higher on II trials than on CI trials. This finding raises the question of whether the RT effect reflects a speed-accuracy trade-off rather than conflict adaptation. To address this interpretive concern, we conducted an additional analysis that indicated that the RT advantage for II nonrepetition trials over CI nonrepetition trials remained significant even when accuracy was matched on II and CI trials for a subset of the sample. Although this result alleviates concern about the RT advantage for II trials being simply a speed-accuracy trade-off rather than adaptation per se, this concern remains viable with regard to the analysis of the degree of conflict, as discussed next.

A secondary issue explored in the present experiment was whether the degree of conflict moderated the degree of adaptation, as revealed by the magnitude of the compatibility effect. After accounting for feature integration effects, the magnitude of the compatibility effect for RT was significantly smaller on nonrepetition trials following high-conflict incompatible trials in comparison with low-conflict or no-conflict (compatible) trials. This finding is consistent with tenets of the conflict monitoring theory. ${ }^{3}$ However, compatibility effects in error rates were increased following high-conflict trials, and this difference persisted in the sample matched on II and CI accuracy. Ullsperger et al. (2005) also observed adaptation in the form of a speed-accuracy trade-off using a modified flanker task. They interpreted this pattern as indicating that task context might have an impact on the particular cognitive control adjustments that are made in response to conflict. In the present paradigm, half of the II trials required participants to respond to up or down central arrows. These stimuli were not naturally mapped to up and down response keys, but rather were associated with the left and right keys during a training phase. If participants implemented a global tightening of cognitive control following the occurrence of a high degree of response conflict (i.e., an incompatible high-conflict trial), and this adjustment led generally to speeded responding, then performance on subsequent incompatible trials in which the target was an up or down arrow may have suffered, because more time may have been needed to translate the stimulus into the appropriate left-right response. This explanation remains preliminary. For now, the moderating effect of the degree of conflict on the adaptation effect in RT should be viewed with caution until it can be shown to occur without the concomitant change in error rate.

\section{GENERAL DISCUSSION}

The present study established several theoretically important points. Experiment 1 indicated that a negativepriming-like slowing influences performance on II nonrepetition trials in a standard Eriksen flanker task. Theoretically, this finding suggests an alternative to the repetition priming account's explanation regarding why conflict adaptation effects are not consistently observed on these trials. The alternative view developed in this article (see also Notebaert \& Verguts, 2006; Ullsperger et al., 2005 ) is that conflict adaptation is occurring, but negative priming is at least partially masking the effect. On the basis of this interpretation, we anticipated that a minor alteration in method would significantly alter the pattern of findings on nonrepetition trials by effectively minimizing the impact of negative priming. This idea was tested in Experiment 2 by enlarging the size of the stimulus set from two to four stimuli. The increased size of the stimulus set was implemented so as to eliminate the occurrence of complete reversals on II nonrepetition trials (i.e., the only type of II nonrepetition trial in the present Experiment 1, in Experiment 1 of Mayr et al., 2003, and in the work of Nieuwenhuis et al., 2006), thereby attenuating negative priming. In line with these predictions, strong evidence of conflict adaptation was observed for RT on nonrepetition trials under these conditions. As in Experiment 1, the conflict adaptation pattern also persisted on repetition trials. Most critically, for nonrepetition trials, the compatibility effect was reduced by $55 \%$ when the preceding trial type was incompatible as opposed to compatible.

Although this pattern is entirely consistent with the assumptions of the conflict monitoring theory, it strongly questions the repetition priming account and feature integration account more broadly. In particular, the findings of Experiment 2 substantiate the notion that conflict adaptation effects do indeed involve top-down control adjustments, adjustments that are most likely to be observed under conditions that minimize negative priming. This conclusion echoes that of Ullsperger et al. (2005), who argued that the unique methodological features they implemented (reviewed above) may have served to avoid the potential masking effect produced by inflated RTs on II nonrepetition trials.

In addition to observing the basic conflict adaptation effect on nonrepetition trials, we also observed adaptation in the form of reduced compatibility effects in RT following high- relative to low-conflict or compatible trials. This finding is consistent with the conflict monitoring account's notion that the magnitude of conflict adapta- 
tion should increase as the degree of conflict on trial $n-1$ increases. This issue deserves further inquiry given the concomitant increase in the magnitude of the compatibility effect in error rate following high-conflict trials. Furthermore, the present account questions whether more conflict will always lead to greater observed adaptation. That is, more conflict could also produce stronger inhibition of the competing response on trial $n-1$, resulting in concomitantly greater negative priming, and potentially stronger masking of conflict adaptation on trial $n$.

One finding that is not readily handled by the present account stems from Experiment 2 of Mayr et al. (2003), wherein conflict adaptation was not observed when using a larger stimulus set consisting of left, right, up, and down arrows. In contrast to the present Experiment 2, their study entailed the use of distinct left, right, up, and down response keys. In addition, the task alternated between stimuli requiring left-right responses and those requiring up-down responses. Trial to trial, the stimuli and responses did not overlap, and therefore it is difficult to argue that negative priming masked the conflict adaptation. Ullsperger et al. (2005) offered several explanations for the findings, each relating to the alternating version used by Mayr et al. Expanding on their explanations, the predictable switching may have permitted participants to make cognitive control adjustments prior to stimulus presentation such that the need for more transient adjustments in control was reduced. Recent computational work modeling interactions between the lateral prefrontal cortex and anterior cingulate shows that increases in proactive control are associated with reduced demands on transient control processes (De Pisapia \& Braver, 2006).

Given that the conflict adaptation effect is one of the primary pieces of behavioral evidence supporting tenets of cognitive control theories (e.g., Botvinick et al., 2001), it seems critical that future studies are directed at further clarifying not only the precise conditions under which humans implement sequential adjustments in cognitive control, but also paradigms that can be used to reliably detect such adjustments. The present finding and past findings suggest that the use of a larger stimulus set may be a critical component of such paradigms, particularly in the case of the Eriksen flanker task. Alternative strategies might also be useful to decrease the potential masking effect of negative priming, such as increasing the length of the RSI or ITI. Supporting this idea, we have observed significant conflict adaptation on complete reversal II nonrepetition trials when the RSI is $1,750 \mathrm{msec}$, but not when the RSI is $1,000 \mathrm{msec}$ (Bugg, 2006). This is consistent with past studies that have characterized the decay function associated with negative priming. For instance, negative priming appears to be most pronounced with RSIs of $1,000 \mathrm{msec}$ or less, as were used in Experiment 1 and in Mayr et al. (2003). It appears to dissipate almost completely at an RSI of 2,000 msec (Neill \& Valdes, 1992; Neill \& Westberry, 1987), which approximates the RSI used by Bugg and is briefer than the shortest ITI used by Ullsperger et al. (2005), both of whom found evidence of conflict adaptation on nonrepetition trials. On the other end of the spectrum, work by Notebaert et al. (2006) indicates that adaptation is observed following a 200 -msec RSI, but not following a brief 50-msec RSI, suggesting that there is a minimum amount of time that is needed to successfully implement control adjustments.

To summarize, few effects are process pure (e.g., Lindsay \& Jacoby, 1994), and the conflict adaptation effect appears to be no exception. We contend that the conflict adaptation effect reflects the dynamic interplay among at least three processes: repetition priming, cognitive control adjustments, and negative priming. The present study has provided initial support for this account by demonstrating the presence of negative priming on critical trial types (e.g., II nonrepetition trials) used to evaluate conflict adaptation (Experiment 1) and by considering all three processes in explaining and predicting the conflict adaptation effect in a second experiment designed to minimize the influence of negative priming. Only an account that emphasizes the notion that negative priming may be working in opposition to conflict monitoring on nonrepetition trials successfully predicts the outcomes of both Experiment 1 and Experiment 2. From a broader perspective, such an account appears fruitful for reconciling the extant literature on conflict adaptation effects in the Eriksen flanker task and other conflict paradigms.

\section{AUTHOR NOTE}

Portions of the data were collected in partial fulfillment of J.M.B.'s doctoral dissertation. The results were presented in part as a poster at the 76th Annual Meeting of the Rocky Mountain Psychological Association, Park City, Utah, and the 14th Annual Meeting of the Cognitive Neuroscience Society, New York. J.M.B. was supported by National Institute on Aging Grant 5T32AG00030 during writing of this article. The author thanks Mark McDaniel for valuable comments on previous versions of this article, and Emily Harmon, Angie Kahrs, Adam Oskvarek, and Becky Tucker for assistance with data collection. The author is grateful to Edward Awh, Matt Botvinick, Michael Kane, and Sander Nieuwenhuis for their thoughtful comments and suggestions on the manuscript. Correspondence concerning this article should be addressed to J. M. Bugg, Department of Psychology, Washington University, Campus Box 1125, One Brookings Drive, St. Louis, MO 63130 (e-mail: jbugg@artsci.wustl.edu).

\section{REFERENCES}

Botvinick, M. M., Braver, T. S., Barch, D. M., Carter, C. S., \& Cohen, J. D. (2001). Conflict monitoring and cognitive control. Psychological Review, 108, 624-652.

Botvinick, M. [M.], Nystrom, L. E., Fissell, K., Carter, C. S., \& Cohen, J. D. (1999). Conflict monitoring versus selection-for-action in anterior cingulate cortex. Nature, 402, 179-181.

BugG, J. M. (2006). Mechanisms of executive control: Behavioral investigations of the conflict adaptation effect. Dissertation Abstracts International, 67(09), 88. (UMI No. 3233326)

De Pisapia, N., \& Braver, T. S. (2006). A model of dual control mechanisms through anterior cingulate and prefrontal cortex interactions. Neurocomputing, 69, 1322-1326.

Egner, T. (2007). Congruency sequence effects and cognitive control. Cognitive, Affective, \& Behavioral Neuroscience, 7, 380-390.

Egner, T., \& Hirsch, J. (2005). The neural correlates and functional integration of cognitive control in a Stroop task. NeuroImage, 24, 539-547.

Eriksen, B. A., \& Eriksen, C. W. (1974). Effects of noise letters upon the identification of a target letter in a nonsearch task. Perception \& Psychophysics, 16, 143-149.

Gratton, G., Coles, M. G. H., \& Donchin, E. (1992). Optimizing the use of information: Strategic control of activation of responses. Journal of Experimental Psychology: General, 121, 480-506.

Hommel, B., Proctor, R. W., \& Vu, K. P. L. (2004). A feature- 
integration account of sequential effects in the Simon task. Psychological Research, 68, 1-17.

Kerns, J. G., Cohen, J. D., MacDonald, A. W., III, Cho, R. Y., Stenger, V. A., \& CARTer, C. S. (2004). Anterior cingulate conflict monitoring and adjustments in control. Science, 303, 1023-1026.

LinDSAY, D. S., \& JACOBY, L. L. (1994). Stroop process dissociations: The relationship between facilitation and interference. Journal of Experimental Psychology: Human Perception \& Performance, 20, 219-234.

MaY, C. P., Kane, M. J., \& HaSher, L. (1995). Determinants of negative priming. Psychological Bulletin, 118, 3-34.

Mayr, U., Awh, E., \& LaURey, P. (2003). Conflict adaptation effects in the absence of executive control. Nature Neuroscience, 6, 450-452.

NeILl, W. T. (1977). Inhibition and facilitation processes in selective attention. Journal of Experimental Psychology: Human Perception \& Performance, 3, 444-450.

NeILL, W. T., \& VALDES, L. A. (1992). Persistence of negative priming: Steady state or decay? Journal of Experimental Psychology: Learning, Memory, \& Cognition, 18, 565-576.

Neill, W. T., \& Westberry, R. L. (1987). Selective attention and the suppression of cognitive noise. Journal of Experimental Psychology: Learning, Memory, \& Cognition, 13, 327-334.

Neumann, E., \& DeSchepper, B. G. (1991). Costs and a benefit of target activation and distractor inhibition in selective attention. Journal of Experimental Psychology: Learning, Memory, \& Cognition, 17, 1136-1145.

Nieuwenhuis, S., Stins, J. F., Posthuma, D., Polderman, T. J. C., Boomsma, D. I., \& DE Geus, E. J. (2006). Accounting for sequential trial effects in the flanker task: Conflict adaptation or associative priming? Memory \& Cognition, 34, 1260-1272.

Notebaert, W., Gevers, W., Verbruggen, F., \& Liefooghe, B. (2006). Top-down and bottom-up sequential modulations of congruency effects. Psychonomic Bulletin \& Review, 13, 112-117.

Notebaert, W., \& Verguts, T. (2006). Stimulus conflict predicts conflict adaptation in a numerical flanker task. Psychonomic Bulletin \& Review, 13, 1078-1084.

Sohn, M. H., \& CARTER, C. S. (2003). Conflict adaptation is independent of stimulus repetition: Evidence for the conflict monitoring model. Manuscript submitted for publication.

Stadler, M. A., \& Hogan, M. E. (1996). Varieties of positive and negative priming. Psychonomic Bulletin \& Review, 3, 87-90.

TIPPER, S. P. (1985). The negative priming effect: Inhibitory effects of ignored primes. Quarterly Journal of Experimental Psychology, 37A, 571-590.
Ullsperger, M., Bylsma, L. M., \& Botvinick, M. M. (2005). The conflict adaptation effect: It's not just priming. Cognitive, Affective, \& Behavioral Neuroscience, 5, 467-472.

Verbruggen, F., Notebaert, W., Liefooghe, B., \& VandierenDONCK, A. (2006). Stimulus- and response-conflict-induced cognitive control in the flanker task. Psychonomic Bulletin \& Review, 13, 328-333.

Wendt, M., Heldmann, M., Munte, T. F., \& Kluwe, R. H. (2007). Disentangling sequential effects of stimulus- and response-related conflict and stimulus-response repetition using brain potentials. Journal of Cognitive Neuroscience, 19, 1104-1112.

WuHr, P., \& ANSORge, U. (2005). Exploring trial-by-trial modulations of the Simon effect. Quarterly Journal of Experimental Psychology, 58A, 705-731.

\section{NOTES}

1. Although slowing on CI trials may not fall under the traditional definition of negative priming, Stadler and Hogan (1996) used the term "negative priming" in a broader sense to describe "any case in which presentation of a stimulus in one instance leads to slowing of performance when that same stimulus is presented at a later time" (p. 87). We use the terminology in a similar vein.

2. I thank Edward Awh for pointing out the importance of evaluating feature integration effects. As he noted, other reports of conflict adaptation in the literature (e.g., Kerns et al., 2004; Sohn \& Carter, 2003) have been subsequently challenged by the feature integration account.

3. In addition to the effect of the degree of conflict on the magnitude of the compatibility effect, there was also a significant main effect of the degree of conflict on the previous trial $\left[F(2,72)=11.86, M S_{\mathrm{e}}=\right.$ $13,285.45]$ such that performance was generally slower following incompatible high-conflict $(M=713)$ as opposed to incompatible lowconflict $(M=641)$ trials. That is, both compatible and incompatible trials were slower following high- ( $M \mathrm{~s}=673$ and 733, respectively) versus low- $(M \mathrm{~s}=538$ and 692, respectively) conflict incompatible trials ( $p<.01$ and $p=.06$, respectively). Although it is not the typical form of conflict adaptation reported herein, the conflict monitoring model does anticipate adaptation effects that take the form of generalized slowing following high conflict. I thank Matt Botvinick for raising this point.

(Manuscript received October 1, 2007; revision accepted for publication May 5, 2008.) 